Journal of Advanced Research in Name
Vol. 00 No. 0, pp. 1-11

November 2013

\title{
On the Order of the Schur Multiplier of a Pair of Finite $p$-Groups
}

\author{
Azam Hokmabadi $^{1}$, Fahimeh Mohammadzadeh ${ }^{1}$, and Behrooz Mashayekhy ${ }^{2, *}$ \\ ${ }^{1}$ Department of Mathematic, Faculty of Sciences, Payame Noor University, \\ 19395-4697 Tehran, Iran. \\ ${ }^{2}$ Department of Pure Mathematics, Center of Excellence in Analysis on Al- \\ gebraic Structures, Ferdowsi University of Mashhad, P. O. Box 1159-91775, \\ Mashhad, Iran.
}

\begin{abstract}
In 1998, G. Ellis defined the Schur multiplier of a pair $(G, N)$ of groups and mentioned that this notion is a useful tool for studying pairs of groups. In this paper, we characterize the structure of a pair of finite p-groups $(G, N)$ in terms of the order of the Schur multiplier of $(G, N)$ under some conditions.
\end{abstract}

Keywords: Pair of groups, Schur multiplier of a pair, Finite $p$-group.

Mathematics Subject Classification 2010: 20E34, 20D15

\section{Introduction}

A definition for the Schur multiplier of a group $G$ is as the abelian group $M(G)=$ $\left(R \cap F^{\prime}\right) /[R, F]$ in which $F / R$ is a free presentation of $G$. In 1956, J.A. Green [4] showed that the order of the Schur multiplier of a finite $p$-group of order $p^{n}$ is bounded by $p^{\frac{n(n-1)}{2}}$, and hence equals to $p^{\frac{n(n-1)}{2}-t}$, for some non-negative integer $t$. Ya.G. Berkovich [1], X. Zhou [13], G. Ellis [3], P. Niroomand [9,10] and E. Khamseh et al. [6] determined the structure of $G$ for $0 \leq t \leq 6$ by different methods.

A pair of groups $(G, N)$ is a group $G$ with a normal subgroup $N$. In 1998, Ellis [2] defined the Schur multiplier of a pair $(G, N)$ of groups to be the abelian group $M(G, N)$ appearing in the natural exact sequence

$$
\begin{aligned}
H_{3}(G) & \rightarrow H_{3}(G / N) \rightarrow M(G, N) \rightarrow M(G) \rightarrow M(G / N) \\
& \rightarrow N /[N, G] \rightarrow(G)^{a b} \rightarrow(G / N)^{a b} \rightarrow 0
\end{aligned}
$$

${ }^{*}$ Correspondence to: Behrooz Mashayekhy, Department of Pure Mathematics, Center of Excellence in Analysis on Algebraic Structures, Ferdowsi University of Mashhad, P. O. Box 1159-91775, Mashhad, Iran. Email: bmashf@um.ac.ir 
in which $H_{3}(G)$ is the third homology of $G$ with integer coefficients. He [2] mentioned that there are three reasons for considering the Schur multiplier of a pair.

1) It leads to a more systematic treatment of a number of elementary results on the usual multiplier $M(G)$. In several instances this treatment yields sharper results.

2) It is a useful tool for studying pairs of groups.

3) It can provide non-trivial information on the third integral homology of a group.

In general, there is no more clear relation between the Schur multiplier of a pair of groups and the usual Schur multiplier than the exact sequence (1.1). However, if $G$ is a semidirect product of $N$ and $K$ with $N \triangleleft G$, then the Schur multiplier of $(G, N)$ is more available to study. In fact, there is an isomorphism $M(G) \cong M(G, N) \times M(K)$ (see [2, page 356]). Recently, some results have been given about the Schur multiplier of a pair of groups $(G, N)$ when $G$ is a semidirect product of $N$ by a complement $K$ (see $[7,8,11,12])$.

Ellis [2] also gave an upper bound for the order of the Schur multiplier of pairs of finite groups. It is interesting to know for which classes of pairs of groups the structure of the pair $(G, N)$ can be completely described in terms of the order of $M(G, N)$. In 2006, Salemkar, Moghaddam and Saeedi [12] tried to answer to this question for a pair of finite $p$-groups and proved the following theorem.

Theorem 1.1. ( [12] ). Let $(G, N)$ be a pair of groups and $K$ be a complement of $N$ in $G$ with $|N|=p^{n}$ and $|K|=p^{m}$. Then the following statements hold:

(i) $|M(G, N)| \leq p^{\frac{1}{2} n(2 m+n-1)}$;

(ii) If $G$ is abelian, $N$ is elementary abelian and $|M(G, N)|=p^{\frac{1}{2} n(2 m+n-1)}$, then $G$ is elementary abelian;

(iii) If the pair $(G, N)$ is non-capable and $|M(G, N)|=p^{\frac{1}{2} n(2 m+n-1)-1}$, then $G \cong \mathbf{Z}_{p^{2}}$.

In this paper we extend the above theorem and characterize the structure of the pair $(G, N)$ of finite $p$-groups in terms of the order of $M(G, N)$ in more cases. Let $(G, N)$ be a pair of finite $p$-groups and $K$ be a complement of $N$ in $G$. In other words, $G$ is a semidirect product of $N$ and $K$. Let $|N|=p^{n},|K|=p^{m}$ and $|M(G, N)|=$ $p^{\frac{1}{2} n(2 m+n-1)-t}$. Then we prove $t=0$ if and only if $N$ is trivial or $(G, N)$ is a pair of elementary abelian $p$-groups. Also, we determine the pair $(G, N)$, for $t=1,2,3$. The main results of this paper are somehow generalizations of the results of [1], [13] and [3] to the pair of finite $p$-groups.

\section{Preliminaries}

Let $(G, N)$ be a pair of groups. We recall that a relative central extension of the pair $(G, N)$ consists of a group homomorphism $\sigma: M \rightarrow G$, together with an action of $G$ on $M$, such that

(i) $\sigma(M)=N$;

(ii) $\sigma\left(m^{g}\right)=g^{-1} \sigma(m) g$, for all $g \in G, m \in M$;

(iii) $m^{\sigma\left(m_{1}\right)}=m_{1}^{-1} m m_{1}$, for all $m, m_{1} \in M$; 
(iv) $G$ acts trivially on $\operatorname{ker} \sigma$.

The $G$-commutator subgroup of $M$ is defined to be the subgroup $[M, G]$ generated by the $G$-commutators $[m, g]=m^{-1} m^{g}$ for all $g \in G, m \in M$, in which $m^{g}$ is the action of $g$ on $m$, and the $G$-center of $M$ is the central subgroup

$$
Z(M, G)=\left\{m \in M \mid m^{g}=m \text { for all } g \in G\right\} .
$$

Also, the subgroup $Z_{2}(M, G)$ is defined by

$$
\frac{Z_{2}(M, G)}{Z(M, G)}=Z\left(\frac{M}{Z(M, G)}, G\right)
$$

A pair $(G, N)$ is said to be capable if it admits a relative central extension $\sigma: M \rightarrow G$ with $\operatorname{ker} \sigma=Z(M, G)$. Note that a group $G$ is capable precisely when the pair $(G, G)$ is capable.

We call a pair $(G, N)$ an extra special pair of $p$-groups when $Z(N, G)$ and $[N, G]$ are the same subgroups of order $p$. Also, we need to recall the definition of a covering pair.

Definition 2.1. ( [2] ). A relative central extension $\sigma: N^{*} \rightarrow G$ of a pair $(G, N)$ will be called a covering pair if there exists a subgroup $A$ of $N^{*}$ such that

(i) $A \leq Z\left(N^{*}, G\right) \cap\left[N^{*}, G\right]$

(ii) $A \cong M(G, N)$;

(iii) $N \cong N^{*} / A$.

Note that Ellis proved that every pair of finite groups has a covering pair (see [2, Theorem 5.4]). The following theorem plays an important role to prove the main results.

Theorem 2.2. ( [2] ). Let $(G, N)$ be a pair of groups and $K$ be a complement of $N$ in $G$. Then

$$
M(G) \cong M(G, N) \times M(K) .
$$

We recall from [5] that if $G=N \times K$, then

$$
M(G) \cong M(N) \times M(K) \times\left(N^{a b} \otimes K^{a b}\right) .
$$

A useful consequence of this fact is stated in the following.

Corollary 2.3. If $G=N \times K$, then

$$
|M(G, N)|=|M(N)|\left|N^{a b} \otimes K^{a b}\right|
$$

The following theorems will be used in the next section. Here $D$ denotes the dihedral group of order $8, Q$ denotes the quaternion group of order 8 and $E_{1}$ and $E_{2}$ denote the extra special groups of order $p^{3}$ with odd exponent $p$ and $p^{2}$, respectively. 
Theorem 2.4. ( [3] ). Let $G$ be a group of order $p^{n}$. Suppose that $|M(G)|=p^{\frac{1}{2} n(n-1)-t}$. Then

(i) $t=0$ if and only if $G$ is elementary abelian ( [1]);

(ii) $t=1$ if and only if $G \cong \mathbf{Z}_{p^{2}}$ or $G \cong E_{1}$ ( [1]);

(iii) $t=2$ if and only if $G \cong \mathbf{Z}_{p} \times \mathbf{Z}_{p^{2}}, G \cong D$ or $G \cong \mathbf{Z}_{p} \times E_{1}$ ( [13]);

(iv) $t=3$ if and only if $G \cong \mathbf{Z}_{p^{3}}, G \cong \mathbf{Z}_{p} \times \mathbf{Z}_{p} \times \mathbf{Z}_{p^{2}}, G \cong Q, G \cong E_{2}, G \cong D \times \mathbf{Z}_{2}$ or $G \cong E_{1} \times \mathbf{Z}_{p} \times \mathbf{Z}_{p}$.

Theorem 2.5. ( [5] ). Let $G \cong \mathbf{Z}_{n_{1}} \times \mathbf{Z}_{n_{2}} \times \ldots \times \mathbf{Z}_{n_{k}}$, where $n_{i+1} \mid n_{i}$ for all $i \in 1,2, \ldots, k-1$ and $k \geq 2$, and let $\mathbf{Z}_{n}^{(m)}$ denote the direct product of $m$ copies of $\mathbf{Z}_{n}$. Then

$$
M(G) \cong \mathbf{Z}_{n_{2}} \times \mathbf{Z}_{n_{3}}^{(2)} \times \ldots \times \mathbf{Z}_{n_{k}}^{(k-1)} .
$$

Theorem 2.6. ( $[2])$. Let $(G, N)$ be a pair of groups such that $N / Z(N, G)$ is finite of prime power order $p^{n}$ and $G / N$ is finite of prime power order $p^{m}$. Then

$$
|M(G, N)||[N, G]| \leq p^{n(2 m+n-1) / 2} .
$$

Theorem 2.7. ( [12] ). Let $(G, N)$ be a pair of finite $p$-groups with $G / N$ and $N / Z(N, G)$ of orders $p^{m}$ and $p^{n}$, respectively. If $|[N, G]|=p^{\frac{1}{2} n(2 m+n-1)}$, then either $N / Z(N, G)$ is elementary abelian or the pair $(G / Z(N, G), N / Z(N, G))$ is an extra special pair of finite p-groups.

Theorem 2.8. ( $[12])$. Let $(G, M)$ be a pair of groups with $G / M$ and $M / Z(M, G)$ of orders $p^{m}$ and $p^{n}$, respectively. Suppose $z \in Z_{2}(M, G)-Z(M, G)$ and consider two non-negative integers $\mu(z)$ and $\nu(z)$, where

$$
p^{\mu(z)}=|[G, z]|, p^{\nu(z)}=\left|\frac{G /[G, z]}{Z(G /[G, z]), M /[G, z])}\right| .
$$

Then

(a) $|[M, G]| \leq p^{\frac{1}{2}(\nu(z)(\nu(z)-1)-m(m-1))+\mu(z)} \leq p^{\frac{1}{2} n(2 m+n-1)}$.

(b) Suppose for some non-negative integer $s,|[M, G]|=p^{n(2 m+n-1) / 2-s}$, then the following statements hold:

(i) $|[M / Z(M, G), G / Z(M, G)]| \leq p^{s+1}$. If $|[M / Z(M, G), G / Z(M, G)]|=p^{s+1-k}$ for some $0 \leq k \leq s+1$, then $\exp \left(Z_{2}(M, G) / Z(M, G)\right) \leq p^{k+1}$ and $m+n-1-s \leq \mu(z) \leq$ $m+n-1-s+k$.

(ii) If $\exp \left(Z_{2}(M, G) / Z(M, G)\right) \geq p^{k}$, then $m+n \leq s /(k-1)+k / 2$.

\section{Main Results}

In this section we always assume that $(G, N)$ is a pair of finite $p$-groups such that $K$ is a complement of $N$ in $G$, with $|N|=p^{n}$ and $|K|=p^{m}$, and hence $|M(G, N)|=$ $p^{\frac{1}{2} n(2 m+n-1)-t}$, for some $t \geq 0$.

Salemkar, Moghaddam and Saeedi [12] proved that if $t=0$ and $G$ is abelian and N is elementary abelian, then $G$ is elementary abelian. The first main result of this paper gives a suitable extended version of the above result similar to Berkovich's one [1]. 
Theorem 3.1. With the previous assumptions and notation, $t=0$ if and only if $N$ is trivial or $(G, N)$ is a pair of elementary abelian $p$-groups.

Proof. Using Theorem 2.2, we have $|M(G, N)|=|M(G)| /|M(K)|$. Hence necessity is immediate by Theorems 2.4. For sufficiency, let the relative central extension $\sigma$ : $N^{*} \rightarrow G$ be a covering pair of $(G, N)$. Note that by [2, Theorem 5.4] the finite pair $(G, N)$ has at least one covering pair. Then there exists a subgroup $A$ of $N^{*}$ such that $A \leq Z\left(N^{*}, G\right) \cap\left[N^{*}, G\right], A \cong M(G, N)$ and $N \cong N^{*} / A$. It is easy to see that for any $k \in K$, the map $\varphi_{k}: N^{*} \rightarrow N^{*}$, defined by $\varphi_{k}\left(n^{*}\right)=n^{* k}$, is an automorphism of $N^{*}$. Therefore, using the homomorphism $\psi: K \rightarrow \operatorname{Aut}\left(N^{*}\right)$, given by $\psi(k)=\varphi_{k}$, we can define a semidirect product of $N^{*}$ by $K$, denoted by $G^{*}$. It is straightforward to check that the subgroups $\left[N^{*}, G\right]$ and $Z\left(N^{*}, G\right)$ are contained in $\left[N^{*}, G^{*}\right]$ and $Z\left(N^{*}, G^{*}\right)$, respectively. Then the map $\delta: G^{*} \rightarrow G$, given by $\delta\left(n^{*} k\right)=\sigma\left(n^{*}\right) k$, for $n^{*} \in N^{*}, k \in K$, is an epimorphism with $\operatorname{ker}(\delta)=\operatorname{ker}(\sigma)$. Therefore

$$
\left|\frac{N^{*}}{Z\left(N^{*}, G^{*}\right)}\right| \leq\left|\frac{N^{*}}{Z\left(N^{*}, G\right)}\right| \leq\left|\frac{N^{*}}{A}\right|=|N|=p^{n}
$$

and

$$
\left|\frac{G^{*}}{N^{*}}\right|=\left|\frac{G^{*} / A}{N^{*} / A}\right|=\left|\frac{G}{N}\right|=|K|=p^{m} .
$$

So $\left|\left[N^{*}, G^{*}\right]\right| \leq p^{\frac{1}{2} n(2 m+n-1)}$ by Theorem 2.6. This implies that

$$
p^{\frac{1}{2} n(2 m+n-1)}=|M(G, N)|=|A| \leq\left|\left[N^{*}, G^{*}\right]\right| \leq p^{\frac{1}{2} n(2 m+n-1)} .
$$

Thus $A=\left[N^{*}, G^{*}\right]$. It follows that $N \leq Z(G)$ and we have $G=N \times K$. Then Corollary 2.3 implies that $p^{\frac{1}{2} n(2 m+n-1)} \leq p^{\frac{1}{2} n(n-1)}\left|N^{a b} \otimes K^{a b}\right|$. Hence $p^{m n} \leq\left|N^{a b} \otimes K^{a b}\right| \leq p^{m d(N)}$, where $d(N)$ is the minimum number of generators of $N$. Therefore $n=d(N)$ and hence $N$ is an elementary abelian $p$-group and $\left|N^{a b} \otimes K^{a b}\right|=p^{m n}$. If $n=0$, then $N$ is the trivial subgroup. If $n>0$, then we have $p^{n m}=\left|\mathbf{Z}_{p}^{(n)} \otimes K^{a b}\right|=p^{d\left(K^{a b}\right) n}$ and it follows that $d\left(K^{a b}\right)=m$. Therefore $d(K)=m$ and so $K$ is an elementary abelian $p$-group too. This completes the proof.

Lemma 3.2. Let $(G, N)$ be a pair of $p$-groups such that $[N, G] \neq 1$. Then $Z(N, G) \cap$ $[N, G] \neq 1$.

Proof. Using the fact that $Z(N, G)=Z(G) \cap N$, the result follows.

Salemkar, Moghaddam and Saeedi [12] proved that if $t=1$ and $(G, N)$ is non-capable, then $G \cong \mathbf{Z}_{p^{2}}$. The second main result of this paper gives a vast generalization of the above result similar to Berkovich's one [1].

Theorem 3.3. With the previous assumptions if $t=1$, then one of the following cases holds:

(i) $G \cong N \times K$ where $N \cong \mathbf{Z}_{p^{2}}$ and $K=1$;

(ii) $G \cong N \times K$, where $N \cong \mathbf{Z}_{p}$ and $K$ is any group with $d(K)=m-1$;

(iii) $(G, N)$ is an extra special pair of groups which is capable. 
Proof. Choose $N^{*}, G^{*}$ and $A$ as in the proof of Theorem 3.1. We divide the proof in two cases:

Case 1. Suppose $A \neq Z\left(N^{*}, G^{*}\right)$. Then $\left|N^{*} / Z\left(N^{*}, G^{*}\right)\right|<\left|N^{*} / A\right|=p^{n}$ and $\left|N^{*} / Z\left(N^{*}, G^{*}\right)\right| \leq$ $p^{n-1}$. Thus by Theorem 2.6

$$
p^{\frac{1}{2} n(2 m+n-1)-1}=|A| \leq\left|\left[N^{*}, G^{*}\right]\right| \leq p^{\frac{1}{2}(n-1)(2 m+n-2)} .
$$

This implies that $n+m \leq 2$. Since $t=1$, by Theorem 3.1 we have $n \neq 0$ and $G$ is not an elementary abelian $p$-group. Therefore we have $G=N \cong \mathbf{Z}_{p^{2}}$.

Case 2. Suppose $A=Z\left(N^{*}, G^{*}\right)$. Then $\left|N^{*} / Z\left(G^{*}, N^{*}\right)\right|=p^{n}$. By Theorem 2.6, we have

$$
p^{\frac{1}{2} n(2 m+n-1)-1}=|A| \leq\left|\left[N^{*}, G^{*}\right]\right|=p^{\frac{1}{2} n(2 m+n-1)-s}
$$

for some $s \geq 0$. It follows that $s \leq 1$.

(i) First, assume that $s=0$. Hence Theorem 2.8 implies that $|[N, G]|=p$ and $\exp (Z(N, G))=p$. If $Z(N, G)$ is cyclic, then $[N, G]=Z(N, G)$ has order $p$ by Lemma 3.2 and hence $(G, N)$ is an extra special pair of groups which is capable. If $Z(N, G)$ is not cyclic, then $Z(N, G)=Z_{2}\left(N^{*}, G^{*}\right) / Z\left(N^{*}, G^{*}\right)$ has two distinct subgroups of order $p$. Therefore there exist elements $y_{0}, z_{0} \in Z_{2}\left(N^{*}, G^{*}\right)-Z\left(N^{*}, G^{*}\right)$ such that

$$
\left|<y_{0} Z\left(N^{*}, G^{*}\right)>\right|=\left|<z_{0} Z\left(N^{*}, G^{*}\right)>\right|=p
$$

and $<y_{0} Z\left(N^{*}, G^{*}\right)>\cap<z_{0} Z\left(N^{*}, G^{*}\right)>=1$. Using the proof of Theorem 2.8, we have $\left|\left[G^{*}, y_{0}\right]\right|=\left|\left[G^{*}, z_{0}\right]\right|=p^{m+n-1}$. On the other hand, $\left[G^{*}, y_{0}\right] \cong G / C_{G^{*}}\left(y_{0}\right)$ and $\left[G^{*}, z_{0}\right] \cong G / C_{G^{*}}\left(z_{0}\right)$. So $\left|C_{G^{*}}\left(y_{0}\right)\right|=\left|C_{G^{*}}\left(z_{0}\right)\right|=p$. Thus we have

$$
Z\left(N^{*}, G^{*}\right) \leq C_{G^{*}}\left(y_{0}\right) \cap C_{G^{*}}\left(z_{0}\right)=\left[N^{*}, G^{*}\right] .
$$

This implies that $|[N, G]|=\left|\left[N^{*} / Z\left(G^{*}, N^{*}\right), G^{*} / Z\left(G^{*}, N^{*}\right)\right]\right|=1$ which is a contradiction.

(ii) Now assume that $s=1$, then $A=\left[N^{*}, G^{*}\right]$. This implies that $N \leq Z(G)$. Thus $G \cong N \times K$. Then by Corollary 2.3 we have $|M(G, N)|=|M(N)|\left|N^{a b} \otimes K^{a b}\right|$. Thus $p^{\frac{1}{2} n(2 m+n-1)-1} \leq p^{\frac{1}{2} n(n-1)} \times\left|N^{a b} \otimes K^{a b}\right|$. Hence $p^{m n-1} \leq\left|N^{a b} \otimes K^{a b}\right| \leq p^{m d(N)}$. Therefore $m(n-d(N)) \leq 1$. Then $m=0$ or $n-d(N)=0$ or $m=n-d(N)=1$. If $m=0$, then $G=N \cong \mathbf{Z}_{p^{2}}$ by Theorem 2.4. If $d(N)=n$, then $N \cong \mathbf{Z}_{p}^{(n)}$. Hence $p^{d(K) n}=\left|N^{a b} \otimes K^{a b}\right|=p^{m n-1}$ and $n(m-d(K))=1$. Therefore $n=1$ and $d(K)=$ $m-1$. In other words, $N \cong \mathbf{Z}_{p}$ and $K$ is any group with $d(K)=m-1$. Finally, if $m=n-d(N)=1$, then $K \cong \mathbf{Z}_{p}$ and $N \cong \mathbf{Z}_{p^{2}} \times \mathbf{Z}_{p}^{(n-2)}$ which by Corollary 2.3 and Theorem 2.5 we must have $n=1$ which is a contradiction.

With an additional condition we will be able to state the reverse of the above theorem as follows.

Theorem 3.4. Let $G \cong N \times K$. Then $t=1$ if and only if one of the following conditions holds: 
(i) $N \cong \mathbf{Z}_{p^{2}}$ and $K=1$;

(ii) $N \cong \mathbf{Z}_{p}$ and $K$ is any group with $d(K)=m-1$;

(iii) $N \cong E_{1}$ and $K=1$.

Proof. By the assumption, $G \cong N \times K$. Hence by Corollary 2.3 we have

$$
|M(G, N)|=|M(N)|\left|N^{a b} \otimes K^{a b}\right| .
$$

Necessity is clear by the above equality. For sufficiency, first we suppose that $N$ is an elementary abelian $p$-group. Then $N \cong \mathbf{Z}_{p^{n}}$ and $\left|N^{a b} \otimes K^{a b}\right|=|M(G, N)| /|M(N)|=$ $p^{m n-1}$. On the other hand, we have $\left|N^{a b} \otimes K^{a b}\right|=\left|\mathbf{Z}_{p}^{(n)} \otimes K^{a b}\right|=p^{n d(K)}$. Therefore $m n-1=n d(K)$ and so $n(m-d(K))=1$. This implies that $N \cong \mathbf{Z}_{p}$ and $d(K)=m-1$.

Now suppose that $N$ is not elementary abelian. Then using (3.1) we have

$$
|M(G, N)|<p^{\frac{1}{2} n(n-1)}|K|^{d(N)} \leq p^{\frac{1}{2} n(n-1)+m d(N)} .
$$

Hence $t=1$ implies that $m(n-d(N))=0$. But $n \neq d(N)$. Therefore $m=0$ and $|M(G, N)|=|M(N)|=p^{\frac{1}{2} n(n-1)-1}$. Hence by Theorem 2.4 we have $K=1$ and $N \cong \mathbf{Z}_{p^{2}}$ or $N \cong E_{1}$. This completes the proof.

The two following theorems determine the pair $(G, N)$, for $t=2$.

Theorem 3.5. . If $t=2$ and $K$ is not a normal subgroup of $G$, then one of the following cases holds:

(i) $G \cong D$ and $N \cong \mathbf{Z}_{4}$;

(ii) $G \cong E_{2}$ and $N \cong \mathbf{Z}_{p}$;

(iii) $(G, N)$ is an extra special pair of groups which is capable;

(iv) $(G, N)$ is a pair of groups with cyclic center and $|Z(G, N)|=p$ and $|[N, G]|=p^{2}$;

(v) $(G, N)$ is a pair of groups with $\exp (Z(G, N))=p^{2}$ and $|[N, G]|=p$.

Proof. Choose $N^{*}, G^{*}$ and $A$ as in Theorem 3.1. Now consider two cases:

Case 1. Suppose $A \neq Z\left(G^{*}, N^{*}\right)$. Then similar to Theorem 3.3 one can show that $n+m \leq 3$. Since $K$ is not normal, we have $m, n \neq 0$.

If $m=1$ and $n=2$, then $|M(G)|=|M(G, N)|$. But $|M(G)|=p^{3-t^{\prime}}$ for some $t^{\prime} \geq 0$ and $|M(G, N)|=p$. Therefore Theorem 2.4 implies that $G=D=\mathbf{Z}_{4} \rtimes \mathbf{Z}_{2}$.

If $m=2$ and $n=1$, then $K=\mathbf{Z}_{p^{2}}$ or $\mathbf{Z}_{p} \times \mathbf{Z}_{p}$. In the first case we have $|M(G)|=$ $|M(G, N)|$. But $|M(G)|=p^{3-t^{\prime}}$ for some $t^{\prime} \geq 0$. Therefore Theorem 2.4 implies that $G=E_{2}$. In the second case we have $|M(G)|=p|M(G, N)|$. Now by Theorem 2.4 we get a contradiction. The other cases do not happen.

Case 2. Suppose $A \cong Z\left(N^{*}, G^{*}\right)$. Then by Theorem 2.6, we have

$$
p^{1 / 2 n(2 m+n-1)-2}=|A| \leq\left|\left[N^{*}, G^{*}\right]\right|=p^{1 / 2 n(2 m+n-1)-s},
$$

for some $s \geq 0$. It follows that $s \leq 2$.

Let $s=0$. Then similar to the proof of Theorem 3.3 (i), we can show that $(G, N)$ is an 
extra special pair of groups which is capable.

Let $s=1$. Then by Theorem $2.8,|[N, G]| \leq p^{2}$ and $\exp (Z(G, N))=p$. If $|[N, G]|=p^{2}$ and $Z(G, N)$ is cyclic, then $|Z(G, N)|=p$ and if $Z(G, N)$ is not cyclic, then similar to the proof of Theorem 3.3 (i) we can show that $Z\left(G^{*}, N^{*}\right)=1$ which is a contradiction. Now suppose that $|[N, G]|=p$. Then by Theorem $2.8, \exp (Z(G, N)) \leq p^{2}$.

If $\exp (Z(G, N))=p$ and $Z(G, N)$ is cyclic, then $(G, N)$ is an extra special pair of groups which is capable. If $\exp (Z(G, N))=p$ and $Z(G, N)$ is not cyclic, then using a similar method to Theorem 3.3 (i), we get a contradiction.

Also $(G, N)$ may be pair of groups with $\exp (Z(G, N))=p^{2}$ and $|[N, G]|=p$.

Let $s=2$. Then $A=\left[N^{*}, G^{*}\right]$. It follows that $N \leq Z(G)$ and we have $G=N \times K$ which is a contradiction.

The next result is somehow a generalization of the Zhou's one [13] to the pair of finite $p$-groups. In this theorem, we suppose that $K$ is normal.

Theorem 3.6. Let $G \cong N \times K$. Then $t=2$ if and only if one of the following conditions holds:

(i) $N \cong \mathbf{Z}_{p} \times \mathbf{Z}_{p^{2}}$ and $K=1$;

(ii) $N \cong D$ and $K=1$;

(iii) $N \cong \mathbf{Z}_{p} \times E_{1}$ and $K=1$;

(iv) $N \cong \mathbf{Z}_{p^{2}}$ and $K \cong \mathbf{Z}_{p}$;

(v) $N \cong E_{1}$ and $K \cong \mathbf{Z}_{p}$

(vi) $N \cong \mathbf{Z}_{p} \times \mathbf{Z}_{p}$ and $K$ is any group with $d(K)=m-1$;

(vii) $N \cong \mathbf{Z}_{p}$ and $K$ is any group with $d(K)=m-2$.

Proof. Since $G \cong N \times K$, by Corollary 2.3 the equality (3.1) holds and the necessity follows. For sufficiency, we proceed as in Theorem 3.4. First suppose that $N$ is an elementary abelian $p$-group. Then $\left|N^{a b} \otimes K^{a b}\right|=|M(G, N)| /|M(N)|=p^{m n-2}$. On the other hand, we have $\left|N^{a b} \otimes K^{a b}\right|=p^{n d(K)}$. Hence $m n-2=n d(K)$ and so $n(m-d(K))=$ 2. It follows that $n=1$ or $n=2$. If $n=1$, then $N \cong \mathbf{Z}_{p}$ and $K$ is any group with $d(K)=m-2$. If $n=2$, then $N \cong \mathbf{Z}_{p} \times \mathbf{Z}_{p}$ and $K$ is any group with $d(K)=m-1$.

Now suppose that $N$ is not an elementary abelian $p$-group. Then (3.1) implies that $|M(G, N)|<p^{\frac{1}{2} n(n-1)}|K|^{d(N)} \leq p^{\frac{1}{2} n(n-1)+m d(N)}$. It follows that $m(n-d(N))<2$. Therefore $m=0$ or $m=1$. If $m=0$, then by Theorem 2.4 we have $K=1$ and $N \cong \mathbf{Z}_{p} \times \mathbf{Z}_{p^{2}}$ or $N \cong D$ or $N \cong \mathbf{Z}_{p} \times E_{1}$. If $m=1$, then $K \cong \mathbf{Z}_{p}$ and $d(N)=n-1$. So $\left|N^{a b} \otimes K^{a b}\right|=p^{n-1}$. Therefore the equality (3.1) implies that $|M(N)|=p^{\frac{1}{2} n(n-1)-1}$. Now using Theorem 2.4 we have $N \cong \mathbf{Z}_{p^{2}}$ or $N \cong E_{1}$. This completes the proof.

Using Theorem 2.2 of [6] and by a method similar to the proof of Theorem 3.5, we have the following theorem.

Theorem 3.7. If $t=3$ and $K$ is not a normal subgroup of $G$, then one of the following cases holds:

(i) $(G, N)$ is an extra special pair of groups which is capable; 
(ii) $(G, N)$ is a pair of groups with $|Z(G, N)|=p,|[N, G]|=p^{2}$ or $p^{3}$;

(iii) $(G, N)$ is a pair of groups with $|[N, G]|=p$ and $\exp (Z(G, N))=p$ or $p^{2}$ or $p^{3}$;

(iv) $G \cong D \times \mathbf{Z}_{2}$ and $N \cong \mathbf{Z}_{\mathbf{2}}$ or $N \cong \mathbf{Z}_{\mathbf{4}} \times \mathbf{Z}_{\mathbf{2}}$;

(v) $G \cong Q_{8} \times \mathbf{Z}_{2}$ and $|N|=2$ or 4 ;

(vi) $G \cong Q_{16}$ or $G \cong D_{16}$ and $N \cong \mathbf{Z}_{\mathbf{2}}$;

(vii) $|N|=p$ or $p^{2}$ and $G \cong E_{2} \times \mathbf{Z}_{p}, T_{1}, T_{4}, X_{1}, X_{4}, X_{5}$ or $X_{6}(p \neq 2)$;

(viii) $N \cong \mathbf{Z}_{\mathbf{p}}$ and $G \cong X_{4} \times \mathbf{Z}_{p}, T_{4} \times \mathbf{Z}_{p}, T_{2}, T_{3}, X_{2}, X_{3}, X_{4}, X_{8}$ or $X_{9}(p \neq 2)$;

where

$X_{1}=\left\langle a, b, c \mid a^{p^{2}}=b^{p}=c^{p}=1,[a, c]=b,[a, b]=[b, c]=1\right\rangle$,

$X_{2}=\left\langle a, b \mid a^{p^{2}}=b^{p^{2}}=1,[a, b]=a^{p}\right\rangle$,

$X_{3}=\left\langle a, b \mid a^{p^{3}}=b^{p}=1,[a, b]=a^{p^{2}}\right\rangle$,

$X_{4}=\left\langle a, b, c \mid a^{p^{2}}=b^{p}=c^{p}=1,[b, c]=a^{p}\right\rangle$,

$X_{5}=\left\langle a, b, c \mid a^{9}=b^{3}=c^{3}=1,[a, b]=1,[a, c]=b, c^{-1} b=a^{-3} b\right\rangle$,

$\left.X_{6}=\langle a, b, c, d| a^{p}=b^{p}=c^{p}=d^{p}=[c, d]=b,[b, d]=a,[a, b]=a, d\right]=[b, c]=[a, c]=$

$1(p>3)\rangle$,

$X_{7}=\left\langle a, b, c \mid a^{p^{2}}=b^{p}=c^{p}=1,[a, c]=b,[b, c]=1,[a, b]=a^{p}\right\rangle$,

$X_{8}=\left\langle a, b, c \mid a^{p^{2}}=b^{p}=1, c^{p}=a^{p},[a, c]=b,[b, c]=1,[a, b]=a^{p}\right\rangle$,

$X_{9}=\langle a, b, c| a^{p^{2}}=b^{p}=1, c^{p}=a^{\alpha p},[a, c]=b,[b, c]=1,[a, b]=a^{p}(\alpha \neq 0, \neq$ and non residue $\bmod p)\rangle$,

$T_{1}=\left\langle a, b, c \mid a^{4}=b^{2}=c^{2}=1,[a, c]=b,[a, b]=[b, c]=1\right\rangle$,

$T_{2}=\left\langle a, b \mid a^{4}=b^{4}=1,[a, b]=a^{2}\right\rangle$,

$T_{3}=\left\langle a, b \mid a^{8}=b^{2}=1,[a, b]=a^{4}\right\rangle$,

$T_{4}=\left\langle a, b, c \mid a^{4}=b^{2}=c^{2}=1,[b, c]=a^{2},[a, b]=[a, c]=1\right\rangle$.

Finally, our last main result is somehow a generalization of the Ellis' one [3] to the pair of finite $p$-groups.

Theorem 3.8. Let $G \cong N \times K$. Then $t=3$ if and only if one of the following conditions holds:

(i) $N \cong \mathbf{Z}_{p^{3}}$ and $K=1$;

(ii) $N \cong \mathbf{Z}_{p} \times \mathbf{Z}_{p} \times \mathbf{Z}_{p^{2}}$ and $K=1$;

(iii) $N \cong Q$ and $K=1$;

(iv) $N \cong E_{2}$ and $K=1$;

(v) $N \cong D \times \mathbf{Z}_{2}$ and $K=1$;

(vi) $N \cong E_{1} \times \mathbf{Z}_{p} \times \mathbf{Z}_{p}$ and $K=1$;

(vii) $N=K \cong \mathbf{Z}_{p^{2}}$;

(viii) $N \cong \mathbf{Z}_{p^{2}} \times \mathbf{Z}_{p}$ and $K \cong \mathbf{Z}_{p}$;

(ix) $N \cong D$ and $K \cong \mathbf{Z}_{p}$;

$(x) N \cong \mathbf{Z}_{p} \times E_{1}$ and $K \cong \mathbf{Z}_{p}$

(xi) $N \cong \mathbf{Z}_{p^{2}}$ and $K \cong \mathbf{Z}_{p} \times \mathbf{Z}_{p}$;

(xii) $N \cong E_{1}$ and $K \cong \mathbf{Z}_{p} \times \mathbf{Z}_{p}$;

(xiii) $N \cong \mathbf{Z}_{p}$ and $K$ is any group with $d(K)=m-3$;

(xiv) $N \cong \mathbf{Z}_{p} \times \mathbf{Z}_{p} \times \mathbf{Z}_{p}$ and $K$ is any group with $d(K)=m-1$. 
Proof. Necessity is straightforward. The proof of sufficiency is similar to the proof of previous theorems. Suppose that $N$ is an elementary abelian $p$-group. Since $t=3$, we have $p^{n d(K)}=\left|N^{a b} \otimes K^{a b}\right|=p^{m n-3}$. This implies that $n(m-d(K))=3$. So $n=1$ or $n=3$. If $n=1$, then $N \cong \mathbf{Z}_{p}$ and $K$ is any group with $d(K)=m-3$. If $n=3$, then $N \cong \mathbf{Z}_{p} \times \mathbf{Z}_{p} \times \mathbf{Z}_{p}$ and $K$ is any group with $d(K)=m-1$.

Now suppose that $N$ is not an elementary abelian $p$-group. Then we have $|M(G, N)|<$ $p^{\frac{1}{2} n(n-1)+m d(N)}$ and so $m(n-d(N)) \leq 2$. This implies that $m=0, m=1$ or $m=2$.

If $m=0$, then by Theorem 2.4 we have $K=1$ and $N \cong \mathbf{Z}_{p^{3}}$ or $N \cong \mathbf{Z}_{p} \times \mathbf{Z}_{p} \times \mathbf{Z}_{p^{2}}$ or $N \cong Q$ or $N \cong E_{2}$ or $N \cong D \times \mathbf{Z}_{2}$ or $N \cong E_{1} \times \mathbf{Z}_{p} \times \mathbf{Z}_{p}$.

If $m=1$, then $K \cong \mathbf{Z}_{p}$ and $d(N)=n-1$ or $d(N)=n-2$. If $d(N)=n-1$, then $\left|N^{a b} \otimes K^{a b}\right|=p^{n-1}$ and so $|M(N)|=|M(G, N)| /\left|N^{a b} \otimes K^{a b}\right|=p^{\frac{1}{2} n(n-1)-2}$. It follows that $N \cong \mathbf{Z}_{p^{2}} \times \mathbf{Z}_{p}$ or $N \cong D$ or $N \cong \mathbf{Z}_{p} \times E_{1}$, by Theorem 2.4. If $d(N)=n-2$, then $|M(N)|=p^{\frac{1}{2} n(n-1)-1}$ and hence $N \cong \mathbf{Z}_{p^{2}}$ or $N \cong E_{1}$ which is a contradiction.

If $m=2$, then $d(N)=n-1$ and $K \cong \mathbf{Z}_{p^{2}}$ or $K \cong \mathbf{Z}_{p} \times \mathbf{Z}_{p}$. If $K \cong \mathbf{Z}_{p} \times \mathbf{Z}_{p}$, then $\left|N^{a b} \otimes K^{a b}\right|=p^{2(n-1)}$ and so $|M(N)|=p^{\frac{1}{2} n(n-1)-1}$. Then $N \cong \mathbf{Z}_{p^{2}}$ or $N \cong E_{1}$. Now suppose $K \cong \mathbf{Z}_{p^{2}}$. Then $N^{a b} \cong \mathbf{Z}_{p}^{(n-1)}$ or $N^{a b} \cong \mathbf{Z}_{p^{2}} \times \mathbf{Z}_{p}^{(n-2)}$. If $N^{a b} \cong \mathbf{Z}_{p}^{(n-1)}$, then $\left|N^{a b} \otimes K^{a b}\right|=p^{n-1}$ and so $|M(N)|=p^{\frac{1}{2}\left(n^{2}+n-4\right)}$ which is a contradiction. If $N^{a b} \cong \mathbf{Z}_{p^{2}} \times \mathbf{Z}_{p}^{(n-2)}$, then $\left|N^{a b} \otimes K^{a b}\right|=p^{n}$ and hence $|M(N)|=p^{\frac{1}{2}\left(n^{2}+n-6\right)}$ which implies that $n \leq 2$. Therefore $N \cong \mathbf{Z}_{p^{2}}$. This completes the proof.

\section{Acknowledgments}

The authors would like to thank the referee for useful suggestions to improve the present paper.

The first and the second authors were partially supported by a grant from Payame Noor University.

\section{References}

[1] Ya.G. Berkovich. On the order of the commutator subgroup and the Schur multiplier of a finite p-group. J. Algebra, 1991, 144: 269-272, doi: 10.1016/0021-8693(91)90106-I.

[2] G. Ellis. The Schur multiplier of a pair of groups. Appl. Categ. Structures, 1998, 6: 355-371, doi: $10.1023 / \mathrm{A}: 1008652316165$.

[3] G. Ellis. On the Schur multiplier of p-groups. Comm. Algebra, 1999, 27(9): 4173-4178, doi: $10.1018 / 00927879908826689$.

[4] J.A. Green. On the number of outomorphisms of a finite $p$-group. Proc. Roy. Soc. London Ser. A, 1958, 237: 574-581.

[5] G. Karpilovsky. The Schur Multiplier. London Math. Soc. Monographs, New Series 2, 1987.

[6] E. Khamseh, M.R. Moghaddam, F. Saeedi. Classification of finite $p$-groups by their Schur multipliers. Group theory conferance, Mashhad, 2010, 102-105.

[7] M.R. Moghaddam, A. Salemkar, T. Karimi. Some inequalities for the order of the Schur multiplier of a pair of groups. Commun. Algebra, 2008, 36(7): 2481-2486, doi: 10.1080/00927870802070033. 
[8] M.R. Moghaddam, A. Salemkar, H.M. Saany. Some inequlities for the Bear-invariant of a pair of finite groups. Indag. Mathem., N.S., 2007, 18(1): 73-82, doi: 10.1016/S00193577(07)80008-5.

[9] P. Niroomand. Characterizing finite $p$-groups by their Schur multipliers. C. R. Math. Acad. Sci. Paris. Ser. I - 1920, 2012, 350: 867-870, doi: 10.1016/j.crma.2012.10.018.

[10] P. Niroomand. Characterizing finite $p$-groups by their Schur multipliers, $t(G)=5$. available at arXiv: math. Gr/ 1001.4257.

[11] A. Salemkar, M.R. Moghaddam, K. Chiti. Some properties on the Schur multiplier of a pair of groups. J. Algebra, 2007, 312: 1-8, doi: 10.1016/j.algebra-2006.11.047.

[12] A. Salemkar, M. R. Moghaddam and F. Saeedi. The commutator subgroup and Schur multiplier of finite $p$-groups. J. Aust. Math. Soc., 2006, 81: 1-9, doi: 10.1017/S1446788700014580.

[13] X. Zhou. On the order of Schur multipliers of finite p-groups. Comm. Algebra, 1994, 22(1): 1-8, doi: 10.1080/00927879408824827. 